\title{
ALGEBRO-GEOMETRIC APPROACH TO THE ERNST EQUATION \\ I. MATHEMATICAL PRELIMINARIES
}

\author{
O. RICHTER and C. KLEIN \\ Max-Planck-Society, Research Unit 'Theory of Gravitation' at the FSU Jena \\ Max-Wien-Platz 1, 07743 Jena, Germany \\ E-mail: oor@gravi.physik.uni-jena.de
}

1. Introduction. It is well known that methods of algebraic geometry and, in particular, Riemann surface techniques are well suited for the solution of nonlinear integrable equations. For instance, for nonlinear evolution equations, so called "finite gap" solutions have been found by the help of these methods. In 1989 Korotkin [9] succeeded in applying these techniques to the Ernst equation, which is equivalent to Einstein's vacuum equation for axisymmetric stationary fields. But, the Ernst equation is not an evolution equation and, due to this fact, one is in this case usually confronted with boundary value problems which have not been considered there.

On the other hand, Neugebauer and Meinel [10] were able to transform the boundary value problem for the rigidly rotating disk of dust into a scalar Riemann-Hilbert problem on a hyperelliptic Riemann surface and gave the solution to this problem in terms of theta functions. The methods they used were suited to the particular problem and one may ask to which extent algebro-geometric methods are useful for the solution of boundary value problems of the Ernst equation. In order to tackle this problem one should at first develop the Riemann-Hilbert technique on Riemann surfaces in detail and then apply this method in order to find solutions to the Ernst equation.

The first of these two papers is devoted to the brief introduction into Riemann surface techniques (for a more detailed exposition see the cited literature). The second paper shows how the developed methods apply to the Ernst equation.

2. Riemann surfaces and abelian differentials. First we give the following

Definition. A compact Riemann surface $\Sigma_{g}$ of genus $g$ is a non-singular surface in $\mathbb{C}^{2}$ given by an equation of the form

1991 Mathematics Subject Classification: 83C20, 83C55.

The paper is in final form and no version of it will be published elsewhere. 


$$
f(K, \mu)=\mu^{n}+a_{1}(K) \mu^{n-1}+\cdots+a_{n}(K)=0,
$$

with $a_{i}$ being polynomials in $K$.

The term non-singular means that

$$
\operatorname{grad} f=\left(\frac{\partial f}{\partial K}, \frac{\partial f}{\partial \mu}\right) \neq 0 .
$$

We call a Riemann surface hyperelliptic if $f$ is of the form

$$
f(K, \mu)=\mu^{2}-P_{n}(K)=\prod_{i=1}^{n}\left(K-E_{i}\right),
$$

with $P_{n}(K)$ being a polynomial without multiple roots. The points $\left(E_{i}, 0\right)$ (or simply $\left.E_{i}\right)$ are the branch points of the hyperelliptic surface. If the branch points are either real or complex conjugated one speaks of a real surface. There is an interesting structure theorem

ThEOREM 1. A hyperelliptic Riemann surface of a function of the form $\mu^{2}=P_{n}(K)$ is diffeomorphic to a sphere with $g$ handles where $n=2 g+1$ or $n=2 g+2$.

For $\Sigma_{g}$ being hyperelliptic we define the hyperelliptic involution $\sigma$ by

$$
\sigma: \Sigma_{g} \ni P=(K, \mu) \rightarrow \sigma(P) \equiv P^{\sigma}=(K,-\mu),
$$

i.e. $\sigma$ interchanges the two sheets of a hyperelliptic Riemann surface. If $\Sigma_{g}$ is in addition real then we may define a further (antiholomorphic) involution $\tau$ by

$$
\tau: \Sigma_{g} \ni P=(K, \mu) \rightarrow \tau(P) \equiv \bar{P}=(\bar{K}, \bar{\mu}),
$$

i.e. real hyperelliptic Riemann surfaces allow for an (at least) four dimensional (Abelian) automorphism group $\operatorname{Aut}\left(\Sigma_{g}\right)$ with generators $\{\sigma, \tau\}$.

For the physical application we have in mind, the structure of branch points of the real hyperelliptic Riemann surface, denoted by $\mathcal{L}_{H}$, is given by

$$
f(K, \mu)=\mu^{2}-(K+\mathrm{i} z)(K-\mathrm{i} \bar{z}) \prod_{i=1}^{g}\left(K-E_{i}\right)\left(K-F_{i}\right),
$$

with either $E_{i}, F_{i} \in \mathbb{R}$ or $E_{i}=\bar{F}_{i}$ and $z=\rho+\mathrm{i} \zeta$. We mention that the above equation has two varying branch points $z$ and $\bar{z}$, representing a family of Riemann surfaces. We will return to the mathematical problems related to this property later.

Let us now introduce a basis for the homology of $\Sigma_{g}$. Because $\Sigma_{g}$ is topologically a sphere with $g$ handles it is a connected, $2 g+1$-connected manifold and its first homology

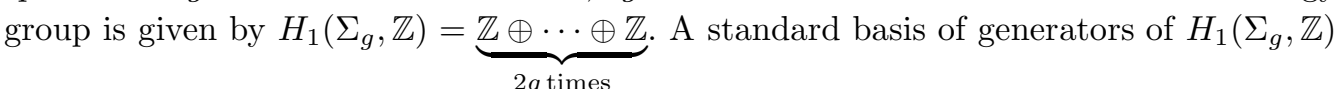
consists of $g$ pairs of onecycles $\left(a_{1}, b_{1}\right), \ldots,\left(a_{g}, b_{g}\right)$ where a pair $\left(a_{i}, b_{i}\right)$ encircles the $i$-th handle (or surrounds the $i$-th hole) so that $a_{i}$ intersects $b_{i}$ orthogonally. The mathematical notion for the statement "one circle intersects another" is the notion of an intersection number. In the figure above we show for two cycles $\gamma_{1}$ and $\gamma_{2}$ under which circumstance the intersection number $\gamma_{1} \cdot \gamma_{2}$ is +1 or -1 . A canonical basis of $H_{1}\left(\Sigma_{g}, \mathbb{Z}\right)$ is now defined as a set of closed curves with intersection numbers

$$
a_{i} \cdot a_{j}=b_{i} \cdot b_{j}=0
$$



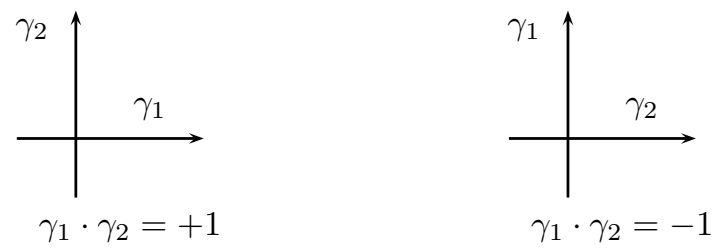

Figure 1: The orientation dependence of the intersection numbers

$$
a_{i} \cdot b_{j}=-b_{i} \cdot a_{j}=\delta_{i j} .
$$

Canonical bases are transformed into each other by integer symplectic transformations, i.e. integer transformations keeping the intersection form

$$
Q=\left(\begin{array}{cc}
0 & I_{g \times g} \\
-I_{g \times g} & 0
\end{array}\right)
$$

invariant. Let us choose for $\mathcal{L}_{H}$ a canonical basis as shown in the figure below. We indicate the parts of the cycles on the upper sheet by solid lines and those on the lower sheet by broken lines.

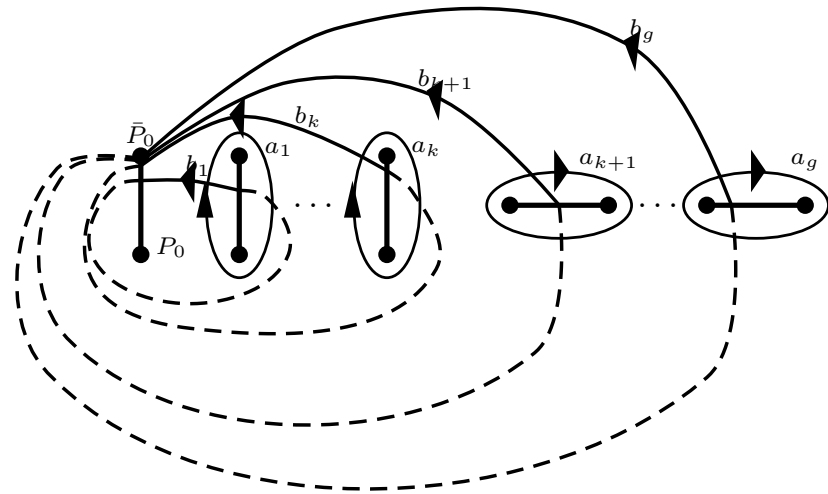

Figure 2: The homology basis for $\mathcal{L}_{H}$

Let us now turn to the differentials on a Riemann surface. A differential (or one form) is called an Abelian differential if it can be written in the neighbourhood of each point in the form

$$
\mathrm{d} \omega=f(K) \mathrm{d} K,
$$

with $f$ being a meromorphic function. Abelian differentials are always closed. They may be decomposed into a sum of differentials of the following three classes:

(i) Abelian differentials of the first kind (holomorphic differentials) $\mathrm{d} \omega_{i}(i=1, \ldots, g)$ : $f(z)$ is a holomorphic function;

(ii) differentials of the second kind $\mathrm{d} \omega_{P}$ : the residue of $f$ at $P$ is zero;

(iii) differentials of the third kind $\mathrm{d} \omega_{P Q}: f$ has two poles with residues \pm 1 .

The "integrated version" of the Abelian differentials are the Abelian integrals on Riemann surfaces which are given by 


$$
I(K, \mu)=\int_{(\xi, \eta)}^{(K, \mu)} P\left(K^{\prime}, \mu^{\prime}\right) \mathrm{d} K^{\prime},
$$

with $P\left(K^{\prime}, \mu^{\prime}\right)$ being a rational function of $K$ and $\mu^{\prime}$ (subject to condition (1)) and $(\xi, \eta) \in \Sigma_{g}$ is fixed. The above classification of Abelian differentials leads immediately to the following classification of Abelian integrals:

(i) Integrals of the first kind: $I(K, \mu)$ is holomorphic on $\Sigma_{g}$.

(ii) Integrals of the second kind: $I(K, \mu)$ has only algebraic poles,

(iii) Integrals of the third kind: $I(K, \mu)$ has two logarithmic poles.

For a closed differential $\mathrm{d} \omega$ we define its periods on the cycles $a_{1}, \ldots, a_{g}, b_{1}, \ldots, b_{g}$ by

$$
\oint_{a_{i}} \mathrm{~d} \omega=A_{i}, \oint_{b_{i}} \mathrm{~d} \omega=B_{i}
$$

$(i=1, \ldots, g)$.

Let us now introduce a normalized basis of holomorphic one forms, defined by the requirement

$$
\oint_{a_{k}} \mathrm{~d} \omega_{j}=2 \pi \mathrm{i} \delta_{j k}
$$

$j, k=1, \ldots, g$. The basis of holomorphic one forms is used to define the so called AbelJacobi mapping

$$
\omega(P)=\left(\int_{P_{0}}^{P} \mathrm{~d} \omega_{1}, \ldots, \int_{P_{0}}^{P} \mathrm{~d} \omega_{g}\right),
$$

with $P_{0}$ - fixed.

Using the normalized holomorphic differentials we define a $g \times g$ matrix - the Riemann matrix $-\Pi$ with entries $\Pi_{j k}$ given by

$$
\Pi_{j k}=\oint_{b_{k}} \mathrm{~d} \omega_{j}
$$

One has

Proposition 2. The matrix $\Pi$ is symmetric and has negative definite real part.

Another important property is stated in the following (with $\mathrm{d} \omega=\left(\mathrm{d} \omega_{1}, \ldots, \mathrm{d} \omega_{g}\right)$ ):

Proposition 3. The vectors $2 \pi \mathrm{i} e^{(i)}=\oint_{a_{i}} \mathrm{~d} \omega$ and $\Pi^{(j)}=\oint_{b_{j}} \mathrm{~d} \omega$ are in $\mathbb{C}^{g}=\mathbb{R}^{2 g}$ linearly independent over the field $\mathbb{R}$.

This proposition allows us to introduce the notion of a characteristic. Any $\boldsymbol{e} \in \mathbb{R}^{2 g}$ can be written uniquely in the form

$$
\boldsymbol{e}=\left(\boldsymbol{\varepsilon}^{\prime}, \boldsymbol{\varepsilon}^{\prime \prime}\right)\left(\begin{array}{c}
2 \pi \mathrm{i} I_{g \times g} \\
\Pi
\end{array}\right)
$$


with $\varepsilon^{\prime}, \varepsilon^{\prime \prime} \in \mathbb{R}^{g}$. We denote by

$$
[\boldsymbol{e}]=\left[\begin{array}{l}
\boldsymbol{\varepsilon}^{\prime} \\
\boldsymbol{\varepsilon}^{\prime \prime}
\end{array}\right]=\left[\begin{array}{l}
\varepsilon_{1}^{\prime}, \ldots, \varepsilon_{g}^{\prime} \\
\varepsilon_{1}^{\prime \prime}, \ldots, \varepsilon_{g}^{\prime \prime}
\end{array}\right]
$$

the characteristic of $\boldsymbol{e}$.

In section 2 we noticed that real hyperelliptic Riemann surfaces admit an antiinvolution $\tau$ generalizing the operation of complex conjugation to Riemann surfaces. Let us now consider the action of $\tau$ on the homology basis, the normalized differentials, and the Riemann matrix of Riemann surfaces of the form (6). One finds for the transformation of the basis elements of $H_{1}\left(\Sigma_{g}\right)$

$$
\begin{aligned}
& \tau\left(a_{i}\right)=-a_{i}, \\
& \tau\left(b_{i}\right)=b_{i}+\sum_{k \neq i} a_{k}, \\
& \tau\left(b_{i}\right)=b_{i}+\sum_{k=1}^{g} a_{k} .
\end{aligned}
$$

Here the second line is true for $b$-cycles whose corresponding $a$-cycle surround two complex conjugated branch points whereas the third line is valid for a $b$-cycle whose corresponding $a$-cycle surrounds two real branch points. For the normalized differentials we have

$$
\tau^{*}\left(\mathrm{~d} \omega_{i}\right)=\overline{\mathrm{d} \omega_{i}},
$$

and the components of the Riemann matrix transform according to

$$
\bar{\Pi}_{i j}=\Pi_{i j}+2 \pi \mathrm{i} \sum_{l \neq i} \delta_{j l},
$$

for ovals $a_{i}$ surrounding two complex conjugated branch points, and

$$
\bar{\Pi}_{i j}=\Pi_{i j}+2 \pi \mathrm{i},
$$

for ovals $a_{i}$ surrounding two real branch points.

3. Picard-Fuchs equations and Gauß-Manin connection. We recall that the spectral parameter of the linear system of the Ernst equation "lives" on a Riemann sphere with variable branch points. This implies that also the real hyperelliptic Riemann surface $\mathcal{L}_{H}$, on which we are looking for solutions has variable branch points. The property that the entries of the Riemann matrix of $\mathcal{L}_{H}$ fulfil certain differential equations - the Picard-Fuchs equations - is due to this fact. These equations turn out to be ordinary differential equations of Fuchsian type, i.e. these equations have only regular singular points. The geometrical interpretation of these equations leads to the concept of a Gauß-Manin connection in the bundle of cohomologies associated with a so called Milnor fibration. For a discussion of these items see [7]. Here we discuss a simple example from [6] which shows already the essential features. Let $\Gamma$ be a closed contour on $S^{2}$ and define

$$
\pi(\lambda)=\oint_{\Gamma} \frac{1}{z(z-\lambda)} \mathrm{d} z .
$$

We have 
and find

$$
\frac{\mathrm{d} \pi(\lambda)}{\mathrm{d} \lambda}=\oint_{\Gamma} \frac{1}{z(z-\lambda)^{2}} \mathrm{~d} z
$$

$$
\begin{aligned}
\lambda \frac{\mathrm{d} \pi(\lambda)}{\mathrm{d} \lambda}+\pi(\lambda) & =\oint_{\Gamma}\left\{\frac{\lambda}{z(z-\lambda)^{2}}+\frac{1}{z(z-\lambda)}\right\} \mathrm{d} z=\oint_{\Gamma} \frac{\mathrm{d} z}{(z-\lambda)^{2}} \\
& =\oint_{\Gamma} \mathrm{d}\left(\frac{1}{\lambda-z}\right)=0,
\end{aligned}
$$

i.e. $\pi(\lambda)$ is a solution to an ordinary homogeneous differential equation of Fuchsian type.

4. Divisors and quasi divisors on compact Riemann surfaces. The RiemannHilbert problem on Riemann surfaces can be stated and solved very elegantly in terms of divisors and quasi divisors.

Definition. A divisor is a formal symbol $\mathfrak{A}$ of the form

$$
\mathfrak{A}=\alpha_{1} P_{1}+\cdots+\alpha_{k} P_{k},
$$

with $P_{i} \in \Sigma_{g}$ and $\alpha_{i} \in \mathbb{Z}$.

The degree of $\mathfrak{A}$ is defined by $\operatorname{deg}(\mathfrak{A})=\alpha_{1}+\cdots+\alpha_{k}$. A divisor is called integral $(\mathfrak{A} \geq 1)$ provided $\alpha_{i} \geq 0 \forall i$. We may introduce a partial ordering on divisors by saying $\mathfrak{A} \geq \mathfrak{B} \Longleftrightarrow \mathfrak{A}-\mathfrak{B} \geq 1$. Let $f$ be a meromorphic function on $\Sigma_{g}$ and let $\mathcal{K}\left(\Sigma_{g}\right)$ denote the set of meromorphic functions on $\Sigma_{g}$. We associate to $f$ a so called principal divisor by $(f)=\sum_{P \in \Sigma_{q}} \operatorname{ord}_{P} f P$ and may define for a divisor $\mathfrak{A}$ a vector space $L(\mathfrak{A})$ by $L(\mathfrak{A}) \doteq$ $\left\{f \in \mathcal{K}\left(\Sigma_{g}\right) \mid(f) \geq \mathfrak{A}\right\}$. The Abel-Jacobi mapping introduced above can be extended to divisors as follows. Let $\mathfrak{A}=\sum_{i} \alpha_{i} P_{i}$ be a divisor and $P_{0} \in \Sigma_{g}$ be fixed. We define

$$
\omega(\mathfrak{A}) \equiv\left(\sum_{i} \alpha_{i} \int_{P_{0}}^{P_{i}} \mathrm{~d} \omega_{1}, \ldots, \sum_{i} \alpha_{i} \int_{P_{0}}^{P_{i}} \mathrm{~d} \omega_{g}\right) .
$$

Definition. A quasi divisor on $\Sigma_{g}$ is a formal symbol

$$
\mathfrak{A}=n_{1} P_{1}+n_{2} P_{2}+\cdots+n_{k} P_{k},
$$

with $P_{i} \in \Sigma_{g}$ and $n_{i} \in \mathbb{R}$.

5. Theta functions associated with a Riemann surface. With the above introduced homology and cohomology basis we may define the building block for the construction of meromorphic functions, the theta function $\Theta(z, \Pi)$ with half integer characteristic associated to a given Riemann surface $\Sigma_{g}$ with homology basis $\left(a_{1} \ldots, a_{g}, b_{1}, \ldots, b_{g}\right)$.

Definition. The Riemann theta function with half integer characteristic $\left[\begin{array}{l}\varepsilon^{\prime} \\ \varepsilon^{\prime \prime}\end{array}\right]$ is given by

$$
\Theta\left[\begin{array}{c}
\varepsilon^{\prime} \\
\varepsilon^{\prime \prime}
\end{array}\right](z, \Pi)=\sum_{N \in \mathbb{Z}^{g}} \exp \left\{\frac{1}{2}\left\langle\Pi\left(N+\varepsilon^{\prime}\right), N+\varepsilon^{\prime}\right\rangle+\left\langle z+2 \pi \mathrm{i} \varepsilon^{\prime \prime}, N+\varepsilon^{\prime}\right\rangle\right\},
$$

with $z \in \mathbb{C}^{g}$ and $\varepsilon^{\prime}, \boldsymbol{\varepsilon}^{\prime \prime} \in \frac{1}{2} \mathbb{Z}^{g}$. 
We denote the theta function with characteristic $\left[\begin{array}{l}\mathbf{0} \\ \mathbf{0}\end{array}\right]$ by $\Theta(z, \Pi)$.

Let us now discuss the zero divisor of the theta functions. We have

Proposition 4. Let $\Theta\left[\begin{array}{c}\varepsilon^{\prime} \\ \varepsilon^{\prime \prime}\end{array}\right](z, \Pi)$ be the theta function with characteristic $\left[\begin{array}{c}\varepsilon^{\prime} \\ \varepsilon^{\prime \prime}\end{array}\right]$ associated to $\left(\Sigma_{g},\{a, b\}\right)$ and let $\omega$ be the Abel-Jacobi map. Then the function

$$
\Theta\left[\begin{array}{l}
\varepsilon^{\prime} \\
\varepsilon^{\prime \prime}
\end{array}\right](z, \Pi) \circ \omega
$$

is either identically zero or has precisely $g$ zeroes. Let for this case be $D=P_{1}+\cdots+P_{g}$ the divisor of zeroes. Then we have

$$
\omega(D)=-\Pi \varepsilon^{\prime}-2 \pi \mathrm{i} \varepsilon^{\prime \prime}+\Pi N+2 \pi \mathrm{i} I M-\vec{K},
$$

with $M, N \in \mathbb{Z}^{g}$ and $\vec{K}$ being the vector of Riemann constants with components

$$
K_{j}=\frac{2 \pi \mathrm{i}+\Pi_{j j}}{2}-\frac{1}{2} \sum_{l \neq j} \oint_{a_{l}}\left(\mathrm{~d} \omega_{l}(P) \int_{P_{0}}^{P} \mathrm{~d} \omega_{j}\right),
$$

$(j=1, \ldots, g)$.

Remark 1 . The Riemann vector $\vec{K}$ depends upon the canonical homology class and the base point of the Abel-Jacobi map.

6. The scalar Riemann-Hilbert problem on a Riemann surface. We follow in this section the exposition in [13]. Let on $\Sigma_{g}$ be given a closed, piece wise smooth contour $\Gamma$. Let there on $\Gamma$ be given a divisor $\Lambda=t_{1}+\cdots+t_{r}$ consisting of a finite number of pair wise different points such that $\Gamma \backslash \Lambda$ decomposes into a finite set of connected components $\Gamma_{j}(j=1, \ldots, N)$, each of which is homeomorphic to the interval $(0,1)$. We call $\Gamma_{j}$ a curve of the contour $\Gamma$. Each $\Gamma_{j}$ has a starting and end point, given by two of the points of $\Lambda$. The starting respectively end points of curves may coincide. We define a function

$$
\alpha\left(t, \Gamma_{j}\right)=\left\{\begin{array}{l}
1 \text { if } t \in \Gamma_{j} \\
0 \text { otherwise }
\end{array},\right.
$$

$(j=1, \ldots, N)$, i.e. $\alpha\left(t, \Gamma_{j}\right)$ is only non vanishing when $t \in \Gamma_{j}$. On each curve $\Gamma_{j}$ let there be defined a Höldercontinuous function $G_{j}(t)$, which is finite and nonzero. We denote

$$
G(t)=\sum_{j=1}^{N} \alpha\left(t, \Gamma_{j}\right) G_{j}(t)
$$

$t \in \Gamma \backslash \Lambda$.

Let there be given a divisor $\mathfrak{A}$ of degree $m$, consisting of the points of the divisor $\Lambda$, taken at arbitrary degree. Let on $\Sigma_{g} \backslash \Gamma$ be given another divisor $\mathfrak{B}$ of degree $n$. We now formulate the

Homogeneous scalar Riemann-Hilbert problem: Solve

$$
\chi^{+}(t)=G(t) \chi^{-}(t),
$$

with $(\chi) \in L\left(\mathfrak{A}^{-1} \mathfrak{B}^{-1}\right)$. 
6.1. The solution of the homogeneous Riemann-Hilbert problem. The solution of the Riemann-Hilbert problem proceeds in several steps. First we need an analog of the Cauchy kernel. We will make use of the kernel, presented already by Weierstrass [12]

$$
K(\tau, \nu)=\frac{f(K, \nu)}{(\nu-\mu) f_{\nu}^{\prime}(\tau, \nu)},
$$

with $f(K, \mu)=0$ and $f(\tau, \nu)=0$.

Next we introduce the canonical function $X(P), P \in \Sigma_{g}$. On each curve $\Gamma_{j}$ of $\Gamma$ the logarithm of the above introduced functions $G_{j}(t)$ is well defined. Denoting

$$
\ln G(t)=\sum_{j=1}^{N} \alpha\left(t, \Gamma_{j}\right) \ln G_{j}(t),
$$

we define a piecewise analytic function function, the canonical function, by

$$
X(P)=e^{Y(P)},
$$

with

$$
Y(P)=\frac{1}{2 \pi \mathrm{i}} \int_{\Gamma} \ln G(\tau) \mathrm{d} \omega_{P P_{0}}(\tau)
$$

Here $\mathrm{d} \omega_{P P_{0}}(\tau)$ is the normalized analog of the Cauchy kernel, $P_{0} \notin \Gamma$ and the integration goes over the whole contour $\Gamma$.

We denote by $(X)$ the principal divisor of the canonical function and want to characterize this divisor more in detail. First we investigate the behaviour of $X$ in the neighbourhood of the starting and end points of the curves of the contour. Let $\Gamma_{j}$ be a curve of the contour $\Gamma$ which starts at $t_{k}$ and ends at $t_{l}$ of $\Lambda$. Let $G_{j}\left(t_{k}+0\right)$ respectively $G_{j}\left(t_{k}-0\right)$ denote the limits of $G_{j}(t)$ in the starting respectively end points of $\Gamma_{j}$. Let $x$ be a local parameter in the neighbourhood of $t_{k}$ and $P=P(x)$ a parametric homeomorphism such that $t_{k}=P(0)$. We obtain the following asymptotic expansion for $X(P)$ in the neighbourhood of $t_{k}$

$$
X[P(x)]=X_{k}(x) \exp \left\{\frac{1}{2 \pi \mathrm{i}}\left(\sum_{j}^{\prime \prime} \ln G_{j}\left(t_{k}-0\right)-\sum_{j}^{\prime} \ln G_{j}\left(t_{k}+0\right)\right) \ln x\right\},
$$

where $\left|X_{k}(x)\right|$ is a function of bounded variation for $x \rightarrow 0$. The summation is over all curves beginning at $t_{k}\left(\sum^{\prime}\right)$ respectively ending at $t_{k}\left(\sum^{\prime \prime}\right)$. We denote by $\arg G_{j}\left(t_{k} \pm 0\right)$ the argument of $\ln G_{j}\left(t_{k} \pm 0\right)$, define

$$
\kappa_{k}=\frac{1}{2 \pi}\left(\sum_{j}^{\prime \prime} \arg G_{j}\left(t_{k}-0\right)-\sum_{j}^{\prime} \arg G_{j}\left(t_{k}+0\right)\right),
$$

and obtain for the asymptotical behaviour of $X(P)$ at $t_{k}$

$$
X[P(x)] \simeq x^{\kappa_{k}} .
$$

From the definition of $X(P)$ it follows that on $\Sigma_{g} \backslash \Gamma$ the canonical function is everywhere finite and different from zero, such that we obtain the following form for the principal divisor

$$
(X)=t_{1}^{\kappa_{1}}+t_{2}^{\kappa_{2}}+\cdots+t_{r}^{\kappa_{r}}
$$


with $\kappa_{i}$ being, in general, real numbers, i.e. $(X)$ is a quasi divisor. Taking the integer part of $\kappa_{i}$ and summing up gives the index of $G(t)$

$$
\kappa=\sum_{i=1}^{r}\left[\kappa_{i}\right]
$$

We want to make the following

Remark 2. For $G(t)$ being a real function we have

$$
\kappa(G(t))=0 .
$$

The proof is rather simple. The argument of $\ln (G(t))$ vanishes up to a multiple of $2 \pi \mathrm{i}$. Fixing a sheet on each curve $\Gamma_{j}$ of the contour $\Gamma$ and summing up gives just the desired result.

By the help of the canonical function we are able to give the solution to the homogeneous Riemann-Hilbert problem. We have

$$
\chi(P)=\gamma(P) \exp \left\{\frac{1}{2 \pi \mathrm{i}} \int_{\Gamma} \ln G(\tau) \mathrm{d} \omega_{P P_{0}}(\tau)-\sum_{j=1}^{g} \int_{\tilde{P}_{j}}^{\hat{P}_{j}} \mathrm{~d} \omega_{P P_{0}}(\tau)\right\},
$$

with $\gamma(P) \in \mathcal{K}\left(\Sigma_{g}\right), \tilde{P}_{j}(j=1, \ldots, g)$ - fixed and the points $\hat{P}_{i}$ being defined as solutions of the so called Jacobi inversion problem

$$
\sum_{j=1}^{g} \omega_{k}\left(\hat{P}_{j}\right)=\int_{\Gamma} \ln G(\tau) \mathrm{d} \omega_{k}(\tau)
$$

with

$$
\omega_{k}\left(\hat{P}_{j}\right)=\int_{P_{0}}^{\hat{P}_{j}} \mathrm{~d} \omega_{k}(\tau)
$$

where $Q \in \Sigma_{g}$ is fixed. The second term can be expressed in terms of theta functions and thus we obtain (independent of the chosen characteristic of the theta function)

$\chi(P)=\gamma(P) \frac{\Theta\left(\omega\left(P_{0}\right)-\omega(\tilde{P})-\vec{K}\right) \Theta(\omega(P)-\omega(\hat{P})-\vec{K})}{\Theta\left(\omega\left(P_{0}\right)-\omega(\hat{P})-\vec{K}\right) \Theta(\omega(P)-\omega(\tilde{P})-\vec{K})} \exp \left\{\frac{1}{2 \pi \mathrm{i}} \int_{\Gamma} \ln G(\tau) \mathrm{d} \omega_{P P_{0}}(\tau)\right\}$

with $\hat{P}=\hat{P}_{1}+\cdots+\hat{P}_{g}, \tilde{P}=\tilde{P}_{1}+\cdots+\tilde{P}_{g}, \omega(P)=\left(\omega_{1}(P), \ldots, \omega_{g}(P)\right)$ and $\vec{K}$ being the vector of Riemann constants.

Remark 3. If $\Gamma$ is a closed smooth contour and $\operatorname{deg}(\mathfrak{B})=0$ then the solution to the homogeneous Riemann-Hilbert problem is unique.

\section{References}

[1] E. D. Belokolos, A. I. Bobenko, V.Z. Enol'skii, A. R. Its, and V. B. Matveev, Algebro-Geometric Approach to Nonlinear Integrable Equations, Springer Series in Nonlinear Dynamics, Springer, Berlin, 1994. 
[2] B. A. Dubrovin, Sov. Math. Survey 36, (1981), 11.

[3] B. A. Dubrovin, A. T. Fomenko, and S. P. Novikov, Modern Geometry - Methods and Applications, Part II. The Geometry and Topology of Manifolds, Graduate Texts in Mathematics, Vol. 104, Springer, Berlin, 1985.

[4] H. M. Farkas and I. Kra, Riemann Surfaces, Graduate Texts in Mathematics, Vol. 71, Springer, Berlin 1993.

[5] John D. Fay, Theta functions on Riemann surfaces, Lecture Notes in Mathematics, vol. 352, Springer, New York, 1973.

[6] P. A. Griffiths, Ann. of Math., (2) 90, (1969), 460.

[7] C. Klein and O. Richter, submitted to C. R. Acad. Sci.

[8] L. Koenigsberger, Vorlesungen über die Theorie der hyperelliptischen Integrale, Teubner, Leipzig, 1878.

[9] D. A. Korotkin, Theor. Math. Phys. 77, (1989), 1018.

[10] G. Neugebauer and R. Meinel, Phys. Rev. Lett, 73, (1994), 2166.

[11] H. Stahl, Theorie der Abelschen Funktionen, Teubner, Leipzig, 1896.

[12] K. Weierstrass, Vorlesungen über die Theorie der Abelschen Transcendenten, Math. Werke, Vol. 4, Berlin, 1902.

[13] S. I. Zverovich, Russ. Math. Survey 26, (1971), 117. 